\title{
Mineração
}

\section{Estudo comparativo entre dois sistemas de aeração de coluna de flotação}

\author{
Ronaldo Penna \\ Engenheiro de Minas, MSc, Bunge Fertilizantes S/A. E-mail: ronaldo.penna@bungeferti.com.br \\ M. Lúcia M. Oliveira \\ Professora, Doutora, Depart. de Engenharia de Minas - Universidade Federal de Ouro Preto \\ E-mail: luciamo@uai.com.br \\ George E. S. Valadão \\ Professor, Doutor, Depart. de Engenharia de Minas - Universidade Federal de Minas Gerais. \\ E-mail:gvaladao@demin.ufmg.br \\ Antônio E. C. Peres \\ Professor, Ph.D. ,Depart. de Engenharia Metalúrgica e de Materiais - Universidade Federal de Minas Gerais. \\ E-mail: aecperes@demet.ufmg.br
}

\section{Resumo}

O aumento na demanda de recursos naturais não renováveis e a exaustão de jazimentos com teores dos elementos de interesse mais elevados têm colocado a indústria mineral frente ao desafio de beneficiar minérios mais complexos e tendo de atender às especificações de mercado, que estão cada vez mais restritas. Considerando essa realidade, novos equipamentos e técnicas para a concentração de minérios têm sido desenvolvidos nos últimos anos. Entre esses desenvolvimentos, a flotação em coluna tem-se mostrado adequada para o tratamento de minérios mais complexos, com teores mais baixos e granulometria de liberação mais fina. O desempenho da coluna de flotação está intimamente relacionado com o controle do tamanho e uniformidade de distribuição das bolhas de ar introduzidas em sua base. Em função disso, diversos tipos de sistemas de aeração têm sido disponibilizados no mercado mundial. Esse trabalho foi realizado com o objetivo de comparar os dois tipos de aeradores que têm sido empregados na indústria mineral brasileira atualmente, ou seja, o aerador tipo lanças perfuradas, que utiliza uma mistura água/ar sob pressão, e o de um único orifício na sua extremidade, que utiliza somente ar. Os resultados obtidos mostram que, nas condições usuais de operação das colunas de flotação, a utilização do aerador tipo água-ar acarreta a geração de bolhas com diâmetro médio menor que os obtidos com o aerador do tipo ar, indicando sua utilização nos processos industriais onde o tamanho e a uniformidade das bolhas geradas sejam fatores decisivos para obtenção de um bom desempenho metalúrgico.

Palavras-chave: flotação em coluna, aeradores industriais, tamanho de bolha.

\begin{abstract}
The growing demand for nonrenewable mineral resources and the exhausting of high grade deposits forced the mineral industry to cope with the challenge of processing ores of increasing complexity aiming at producing concentrates meeting specifications that become continuously more strict. Under this scenario, innovative pieces of equipment and techniques for the concentration of ores have been developed in the past years. Among these developments, column flotation proved to be a proper technique for processing low grade ores, presenting a high liberation degree only at fine size ranges. The performance of a flotation column is closely related to the size and distribution uniformity of the air bubbles introduced at its bottom. Consequently several types of sparging systems are available in the world market. This investigation was performed aiming at comparing two types of spargers that are presently utilized in Brazilian concentrators: the standard air/water sparger system and the single nozzle (at the end) sparger, utilizing air only. The results indicated that, under the usual operation conditions of flotation columns, the water/air sparger generates bubbles with smaller mean diameters than those achieved with the air only sparger. The water/air sparger is recommended in industrial applications requiring small bubbles, uniform in size, for enhanced metallurgical performance.
\end{abstract}

Keywords: column flotation, industrial spargers, bubble size. 


\section{Introdução}

O processo de flotação é um método de concentração mineral que utiliza a diferença entre as propriedades de superfície das partículas minerais como meio de separação. Foi desenvolvido no início do século passado e tem sido economicamente utilizado para concentração de minérios complexos, com baixos teores dos minerais de interesse, levando a bons rendimentos metalúrgicos (Kelly \& Spottiswood, 1982). Sua aplicação estende-se a uma grande variedade de minérios com diferentes granulometrias, composições químicas e mineralógicas e, principalmente, diferentes propriedades físico-químicas de superfície dos minerais constituintes.

O crescimento acelerado das demandas por recursos minerais não renováveis tem acarretado a exaustão dos jazimentos mais ricos e mais simples de serem concentrados, colocando a indústria mineral frente ao desafio de tratar minérios cada vez mais complexos, com tamanho de liberação muita fina e com baixos teores. Além disso, devido à globalização da economia mundial, as unidades industriais têm sido obrigadas a produzir concentrados com especificações cada vez mais severas e custos menores. Considerando essa realidade e a importância da flotação como processo de concentração de minerais, têm sido realizados esforços no sentido de desenvolver novas tecnologias e equipamentos, dentre os quais pode ser destacada a coluna de flotação. Esse equipamento, idealizado na década de 60 , teve a sua primeira aplicação industrial no início da década de 80 e vem sendo utilizado, principalmente, na concentração de minérios com granulometria fina e em estágios de limpeza (Wheeler, 1986).

A coluna de flotação apresenta características diferentes das células mecânicas, principalmente no que se refere à capacidade de geração de bolhas pequenas, as condições hidrodinâmicas de baixa turbulência e a possibilidade de eliminação do material hidrofílico arrastado pela espuma. Além disso, o "hold up" de ar na coluna de flotação é significativamente mais elevado que nas células mecânicas. O fluxo de polpa des- cendente, alimentado a aproximadamente dois terços da altura total da coluna, é contactado em contracorrente com o fluxo de ar ascendente, introduzido na sua base através de aeradores, resultando no processo de coleta das partículas hidrofóbicas. O agregado partícula-bolha formado é, então, transportado até a zona de limpeza onde encontra um fluxo de água de lavagem introduzido no topo da coluna.

Considerando que as bolhas de ar são responsáveis pela coleta e transporte das partículas hidrofóbicas da polpa para a espuma, torna-se de fundamental importância a utilização de sistemas de aeração que permitam o controle do tamanho e da uniformidade das bolhas geradas através de ajustes operacionais e da utilização de agentes sulfatantes. Em função disso, as indústrias de fabricação de colunas têm disponibilizado no mercado mundial diversos tipos de sistemas de aeração, que, além de atender a essas exigências, apresentam condições de operação e de manutenção cada vez mais simples.

Um gerador de bolhas eficiente é aquele capaz de gerar bolhas de 0,5 a 2,0 mm de diâmetro, com a velocidade superficial de ar entre 1,0 e 3,0 cm/s e hold up de 15 a $20 \%$. Além disso, deve ter manutenção mecânica e operação fácil e ser produzido com materiais resistentes ao desgaste (Luz et al., 1998). O tamanho médio das bolhas e sua distribuição são responsáveis pela eficiência de coleta e de transporte das partículas. Bolhas pequenas apresentam área superficial elevada favorecendo a cinética de coleta e transporte dos sólidos para um dado volume de ar. Por outro lado, as bolhas de tamanho muito pequeno podem ter uma velocidade de ascensão mais baixa que a velocidade descendente da polpa, levando a perdas de partículas hidrofóbicas no fluxo de material não flotado. Portanto o tamanho médio ideal de bolhas é função do tamanho médio das partículas e varia de 0,5 a 2,0 mm nas colunas de flotação.

Na década de 60, quando a coluna de flotação foi desenvolvida, eram utilizados aeradores internos construídos em materiais porosos, principalmente borracha microperfurada (Wheeler, 1986). Esses aeradores apresentavam como prin- cipal desvantagem os freqüentes entupimentos e a dificuldade de substituição, exigindo a parada da coluna para sua substituição. Os primeiros aeradores externos utilizados nas colunas industriais consistiam basicamente de lanças perfuradas alimentadas com uma mistura de água e ar sob pressão. Esses aeradores apresentavam como principais vantagens a possibilidade de remoção, inspeção e substituição dos injetores com a coluna em operação e a geração de bolhas menores e mais uniformes. Embora esses aeradores tenham representado um significativo avanço tecnológico, são de difícil operação e apresentam entupimentos freqüentes devido à obstrução dos furos das lanças pelas impurezas da água. Em função disso, os aeradores que utilizam a mistura arágua sob pressão vêm entrando em desuso e sendo substituídos por aeradores que utilizam somente ar. Esses aeradores consistem de lanças com um único orifício na sua extremidade para a saída do ar a uma velocidade próxima à do som, dispensando o uso de água. Esse tipo de aerador é de operação mais fácil e apresenta menor probabilidade de entupimento.

No Brasil, a maior parte das colunas industriais de flotação existentes foi projetada para operar utilizando o sistema de geração de bolhas de lanças perfuradas da Cominco Engineering Services LTD (hoje CPTI). Mais recentemente, a partir do desenvolvimento do sistema que utiliza lanças com um único orifício, fabricado pela CPTI e/ou Minnovex Technologies Inc, algumas unidades industriais brasileiras passaram a utilizar esse novo modelo. Entretanto não se tem notícia sobre a realização de um estudo sistemático que permitisse uma análise comparativa do desempenho desses dois sistemas de aeração.

Nesse sentido foi realizado esse trabalho, tendo como principal objetivo comparar os dois tipos de aeradores que têm sido mais utilizados na indústria mineral brasileira, ou seja, o aerador tipo lanças perfuradas, que utiliza uma mistura de água e ar sob pressão, e o de um único orifício na sua extremidade, que utiliza somente ar, frente às variações de alguns parâmetros operacionais e de processo da coluna. 


\section{Metodologia}

Esse trabalho foi realizado em uma coluna de flotação de $0,61 \mathrm{~m}$ de diâmetro e 4,7 $\mathrm{m}$ de altura, de propriedade da Supervisão de Processos do Centro de Desenvolvimento da Tecnologia Nuclear - CDTN, operando no sistema bifásico água e ar em circuito fechado, com recirculação do fluxo de água descendente na alimentação da coluna. Na seção de recuperação dessa coluna estão instalados dois sensores de pressão para determinação dos valores do hold up do ar e visores em acrílico para permitir a observação do tamanho e da distribuição das bolhas geradas. O fluxograma do circuito utilizado está apresentado na Figura 1.

Foram estudados os aeradores tipo água-ar da CPTI e o tipo ar da Minnovex Technologies Inc. $\mathrm{O}$ aerador tipo águaar é constituído de lanças perfuradas com orifícios de $0,9 \mathrm{~mm}$ alimentadas com uma mistura de água e ar sob pressão. Esse modelo apresenta a vantagem de permitir o ajuste do tamanho das bolhas através da atuação na pressão do sistema e na relação entre as vazões de ar e de água. Por outro lado, além de ter uma operação complexa, exigindo equilíbrio entre as pressões e taxas de fluxo do ar e da água, apresenta entupimentos freqüentes dos orifícios das lanças causados pelas impurezas da água de processo utilizada. $\mathrm{O}$ aerador tipo ar consiste de lanças de aço inoxidável com uma polegada de diâmetro e um único orifício de 3,2 mm de diâmetro na extremidade, revestido de material cerâmico, para a saída de ar. A velocidade de saída do ar no furo é próxima à velocidade do som permitindo a geração de bolhas pequenas. O tamanho das bolhas é controlado através do ajuste da vazão e da pressão de operação do ar, atuando em uma válvula de agulha instalada na entrada da lança. Esses aeradores apresentam as vantagens de dificilmente entupirem, terem manutenção mecânica muito simples, tamanho compacto e vida útil longa.

As variáveis de estudo foram a dosagem do reagente espumante Flotanol D14-B, as velocidades superficiais de ar e da polpa descendente e a pressão de trabalho do ar ou do sistema água-ar. Foram considerados como variáveis de resposta os diâmetros das bolhas geradas, calculados através do Drift Flux (Dobby et al., 1986) e do hold up do ar.

A velocidade superficial de um fluxo na coluna $\left(\mathrm{J}_{\mathrm{f}}\right)$ é definida pela relação entre a vazão desse fluxo $\left(\mathrm{Q}_{\mathrm{g}}\right)$ e a área da seção transversal da coluna $\left(\mathrm{A}_{\mathrm{c}}\right)$ e é expressa em $\left(\mathrm{cm}^{3} / \mathrm{s}\right) / \mathrm{cm}^{2} \mathrm{ou} \mathrm{cm} / \mathrm{s}$. A velocidade superficial foi calculada por:

$$
J_{f}=Q_{f} / A_{c}
$$

O drift flux, desenvolvido por Dobby et al. (1986), é um modelo matemático empírico que tem por objetivo estimar o diâmetro médio das bolhas de ar em colunas de flotação, baseado essencialmente nos valores do hold up do ar e nas diversas velocidades das fases presentes na coluna. $\mathrm{O}$ diâmetro das bolhas foi estimado a partir das seguintes equações:

$$
\begin{aligned}
& d_{b}=\left[\frac{18 \mu_{s \ell} \cdot U_{\tau}}{g \cdot \Delta \rho} \cdot\left(1+0,15 \cdot \mathrm{Re}_{p}^{0,687}\right)\right]^{1 / 2} \\
& U_{\tau}=\frac{J_{g}}{\varepsilon_{g} \cdot\left(1-\varepsilon_{g}\right)^{m}}-\frac{\left(J_{g}+J_{\ell}\right)}{\left(1-\varepsilon_{g}\right)^{m}} \\
& m=\left(4,45+18 \cdot d_{b} / d_{c}\right) \cdot \operatorname{Re}_{b}^{-0,1} \text { para } 1<\mathrm{R}_{\mathrm{eb}}<200 \\
& m=4,45 \cdot \operatorname{Re}_{b}^{-0,1} \text { para } 200<\mathrm{R}_{\mathrm{eb}}<500
\end{aligned}
$$

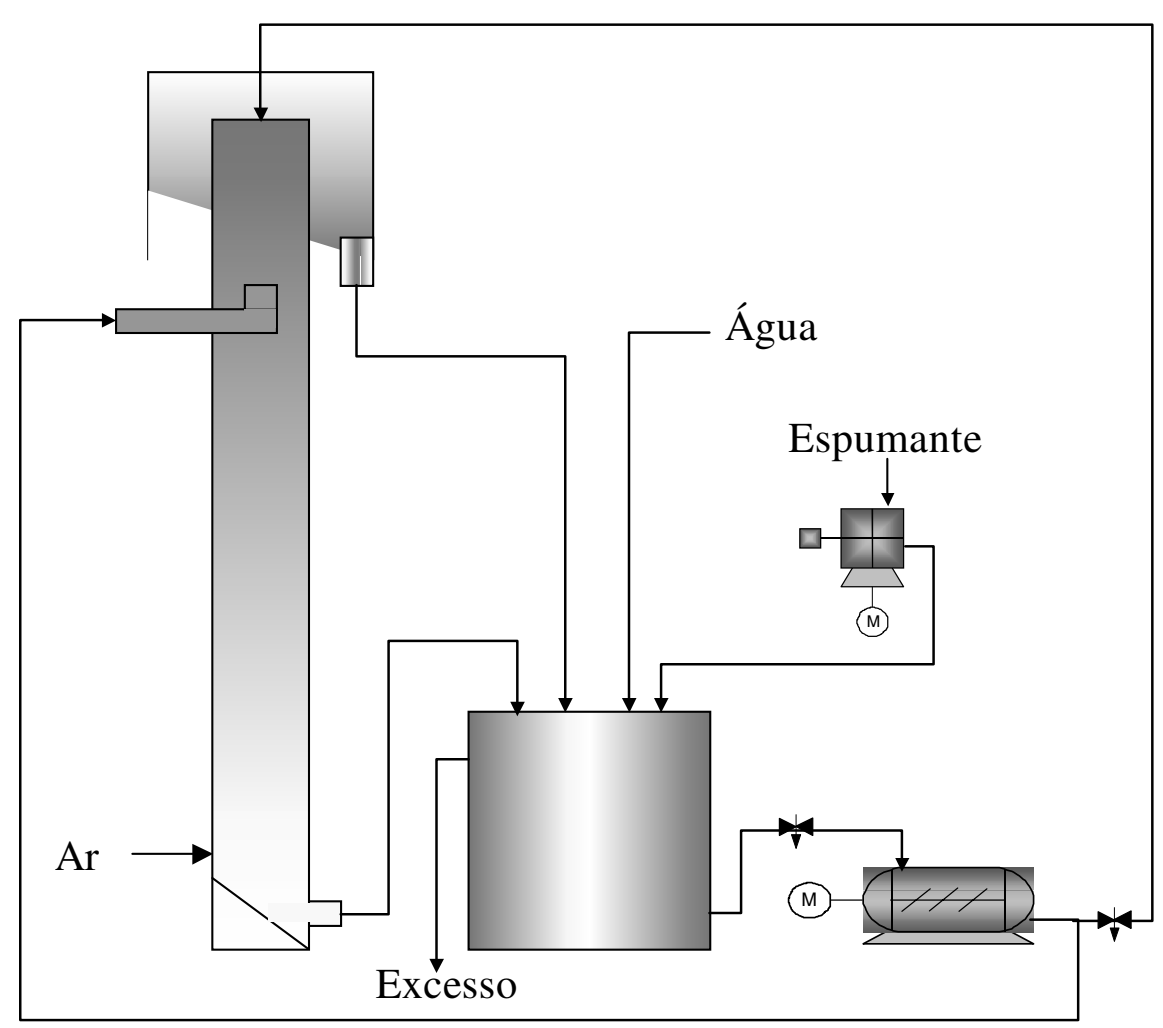

Figura 1 - Fluxograma do circuito utilizado para realização dos testes. 
$\operatorname{Re}_{b}=\frac{d_{b} \cdot U_{\tau} \cdot \rho_{s \ell}}{\mu_{s \ell}}$

onde:

$\mathrm{db}=$ Diâmetro médio da bolha;

$\mathrm{d}_{\mathrm{c}}=$ Diâmetro da coluna;

$\stackrel{\mathrm{U} \tau}{\tau}=$ Velocidade terminal de ascensão das bolhas de ar;

$\mu_{\mathrm{sl}}=$ Viscosidade da polpa;

$\rho_{\mathrm{sl}}=$ Densidade da polpa;

$\mathrm{g}=$ Aceleração da gravidade;

$\mathrm{Re}_{\mathrm{p}}=$ Número de Reynolds das partículas;

$\operatorname{Re}_{\mathrm{b}}^{\mathrm{p}}=$ Número de Reynolds das bolhas;

$\Delta \rho=$ Diferença de densidade entre as

fases polpa e ar;

$\mathrm{J}_{\mathrm{g}}=$ Velocidade superficial do ar;

$\mathrm{J}_{1}^{\mathrm{g}}=$ Velocidade superficial do líquido.

O hold up do ar, definido como a percentagem do volume da coluna ocupado pelo ar, é determinado na seção de recuperação e constitui um parâmetro que depende da vazão de ar, do tamanho das bolhas, da densidade de polpa, do carregamento de sólidos nas bolhas e da velocidade descendente de polpa. Através de sua medida é possível estimar o diâmetro de bolha através de modelos matemáticos (Finch \& Dobby, 1990). O hold up do ar foi calculado pela seguinte equação:

$\varepsilon_{g}=1-\frac{\Delta P}{\rho_{S l} \cdot g \cdot L}$

onde:

$\Delta P=$ Diferença de pressão entre dois pontos da zona de recuperação $(\mathrm{kPa})$;

$\rho_{s l}=$ Densidade da polpa entre esses pontos $\left(\mathrm{g} / \mathrm{cm}^{3}\right)$;

$L=$ Distância entre os pontos das medidas de pressão (m);

$g=$ Aceleração da gravidade $\left(\mathrm{m} / \mathrm{s}^{2}\right)$.

\section{Resultados e discussão}

O desempenho dos aeradores atualmente utilizados nas unidades industriais de flotação em coluna em operação no Brasil, ou seja, tipo água-ar e somente ar com orifício de 3,2 mm de diâmetro, foi comparado através da análise dos valores do hold up do ar e do diâmetro das bolhas frente a variações na dosagem do reagente espumante Flotanol
D14-B, nas velocidades superficiais de ar e da polpa descendente e na pressão de trabalho do ar ou do sistema água-ar. Os resultados obtidos estão apresentados a seguir.

\subsection{Dosagem de espumante}

Uma das importantes vantagens que a coluna de flotação apresenta em relação às células mecanicamente agitadas consiste na redução do arraste hidráulico de partículas hidrofílicas através da utilização de camadas de espuma de alturas elevadas, ou seja, da ordem de 1 a 2 metros. Partículas hidrofílicas e/ ou menos hidrofóbicas que atingem a interface polpa espuma são gradualmente lavadas e retornadas à seção de recuperação aumentando a seletividade do processo. O espumante Flotanol D14-B, eterpolialquilenoglicol, fabricado pela Clariant, é adicionado às colunas com objetivo de produzir bolhas com um tamanho médio adequado à flotação e obter uma maior estabilidade da espuma. Foram realizados testes variando a dosagem de flotanol de 0 a $30 \mathrm{mg} / \mathrm{L}$. Os resultados obtidos estão apresentados nas Figuras 2 e 3 e mostram que, para ambos os aeradores, o hold up do ar foi crescente com o aumento da dosagem de espumante até uma concentração de $20 \mathrm{ppm}$, a partir da qual manteve-se praticamente constante. Utilizando $25 \mathrm{ppm}$ de espumante o tamanho médio das bolhas geradas nos aeradores tipo água-ar $(0,7 \mathrm{~mm})$ foram significativamente menores que aquelas geradas no aerador tipo somente ar (1,2 mm).

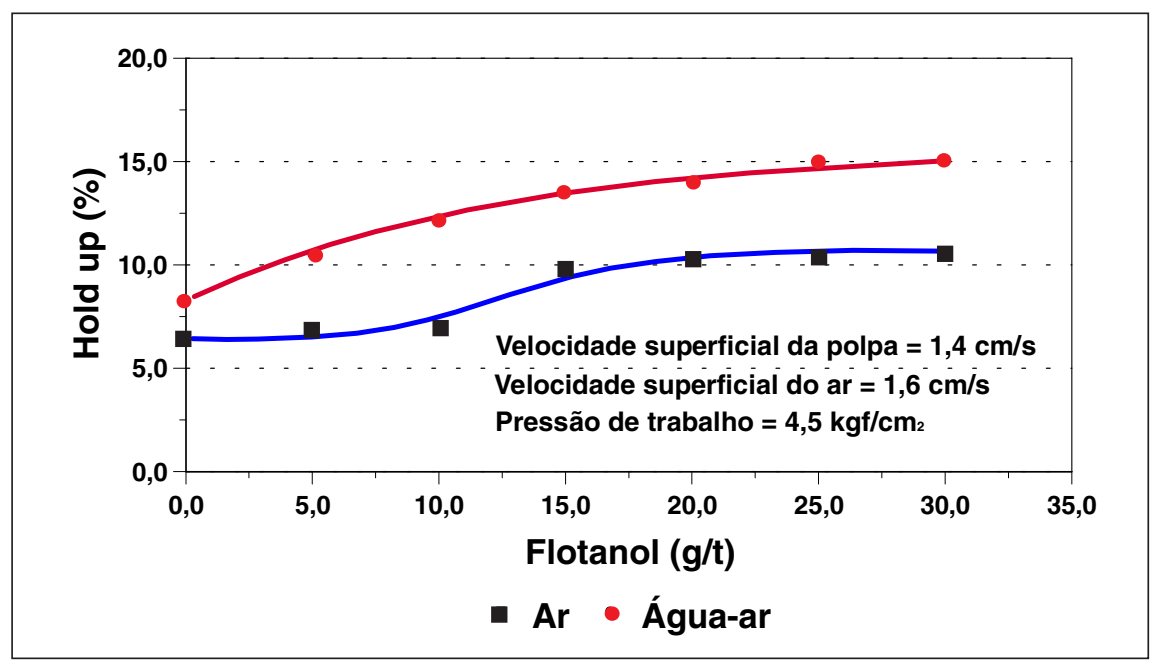

Figura 2 - Efeito da dosagem de espumante sobre o hold up do ar na coluna.

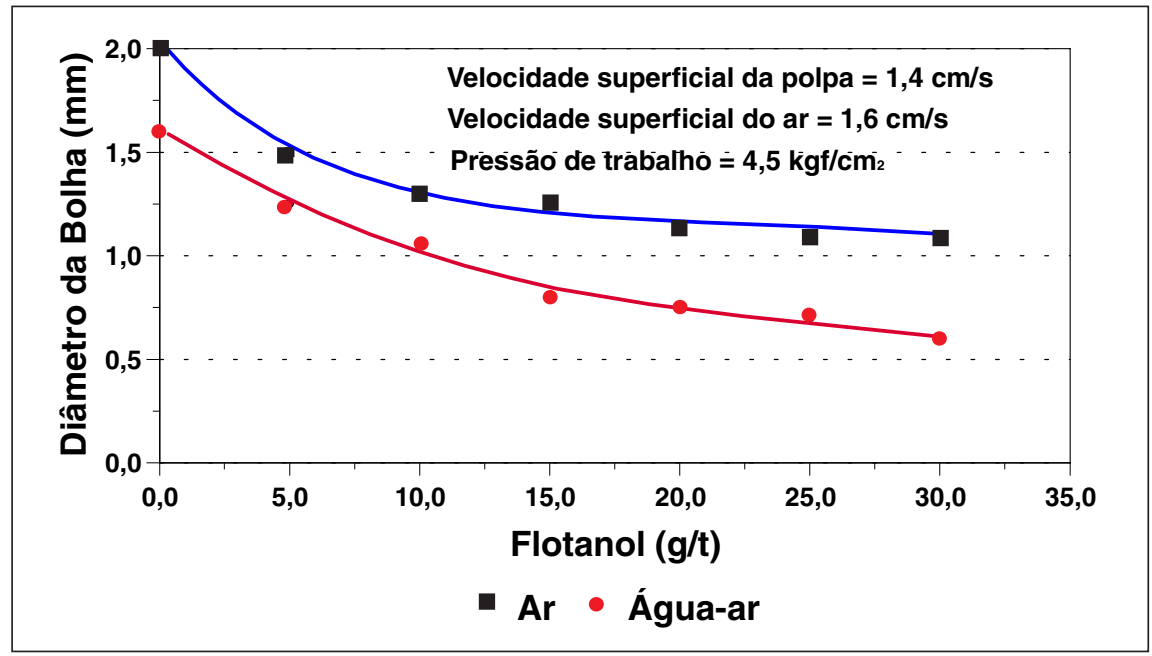

Figura 3 - Efeito da dosagem de espumante sobre o tamanho das bolhas. 


\subsection{Velocidade superficial do ar}

A velocidade superficial do ar é uma das variáveis mais importantes no controle do processo de flotação em coluna tendo um efeito significativo sobre a recuperação do mineral na fração flotada. Isso se deve à influência dessa variável sobre a disponibilidade de bolhas com tamanho e distribuição adequados para a coleta e o transporte das partículas minerais da seção de coleta da coluna para a de limpeza. Para avaliar o efeito desse parâmetro sobre o hold up do ar e o diâmetro médio das bolhas foram realizados testes variando a velocidade superficial do ar de 0,7 a $1,9 \mathrm{~cm} / \mathrm{s}$, utilizando os dois aeradores. Os resultados estão apresentados nas Figuras 4 e 5 e mostram que foram obtidos valores de hold up do ar significativamente superiores e, consequientemente, tamanhos de bolha menores, para o aerador tipo água-ar.

\subsection{Velocidade superficial da polpa}

O fluxo descendente de polpa na seção de recuperação da coluna acarreta um aumento no tempo de retenção das bolhas de ar ascendentes levando a um aumento no hold up e, conseqüentemente, a uma redução no tamanho das bolhas. Para comparar a eficiência dos dois tipos de aeradores, foram realizados testes variando a velocidade superficial do fluxo

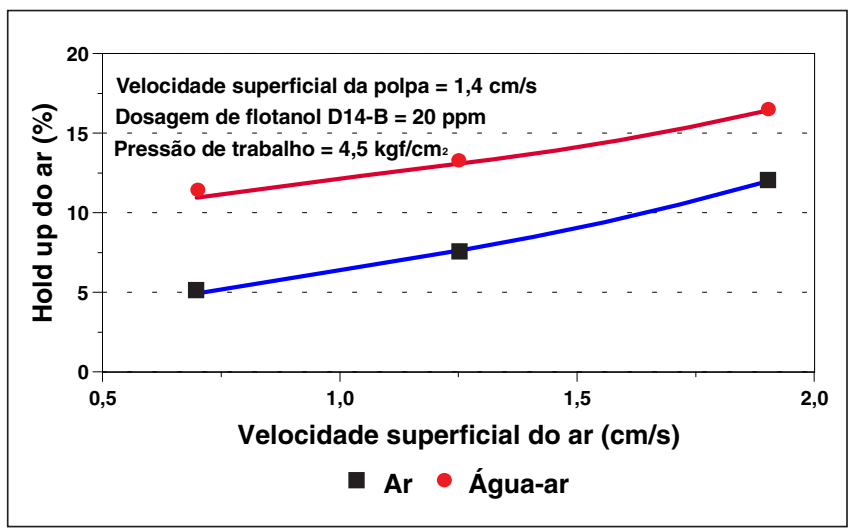

Figura 4 - Efeito da velocidade superficial do ar sobre o hold up na coluna.

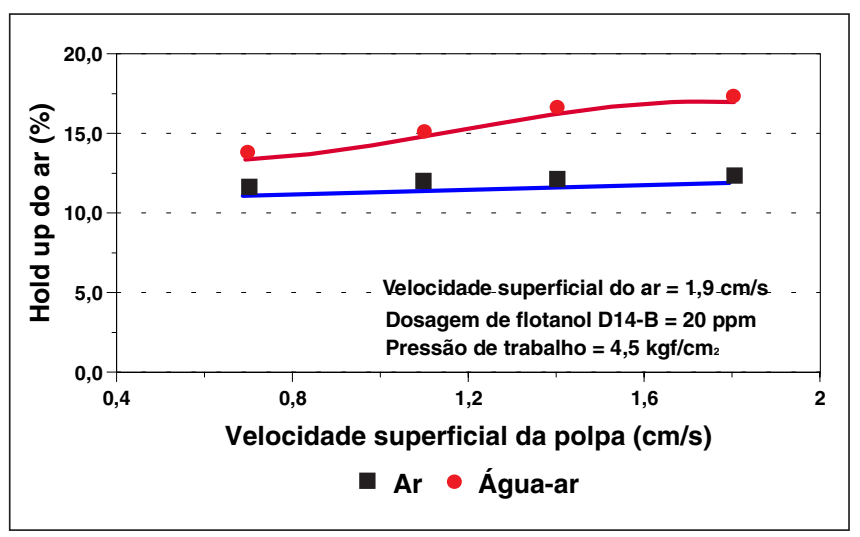

Figura 6 - Efeito da velocidade superficial da polpa sobre o hold up do ar na coluna. descendente de 0,5 a 2,0 cm/s. Os resultados obtidos estão apresentados nas Figuras 6 e 7 e mostram que, utilizando o aerador água-ar, o hold up foi crescente com o aumento da velocidade da polpa, enquanto com o aerador tipo ar mantevese praticamente constante. Isso se deve, provavelmente, à maior turbulência da polpa provocada por esse sistema. Embora ambos aeradores tenham gerado bolhas de tamanho decrescente com o aumento da velocidade da polpa, o aerador água-ar produz bolhas significativamente menores.

\subsection{Pressão de trabalho do ar}

A pressão de trabalho do ar ou do sistema água-ar é uma variável de fundamental importância para garantir a estabilidade da geração das bolhas na coluna de flotação e manter os orifícios dos aeradores desobstruídos. Os resultados obtidos nos testes realizados variando a pressão do ar de 3,8 a 6,2 kgf/ $\mathrm{cm}^{2}$ estão apresentados nas Figuras 8 e 9 . Esses resultados mostram que, para obtenção de níveis mais elevados de hold up do ar, utilizando o aerador tipo ar, foi necessário utilizar pressões superiores às usadas no tipo água-ar. Além disso, em condições idênticas de operação, o aerador tipo água-ar apresentou um tamanho de bolha sistematicamente menor que o do tipo ar.

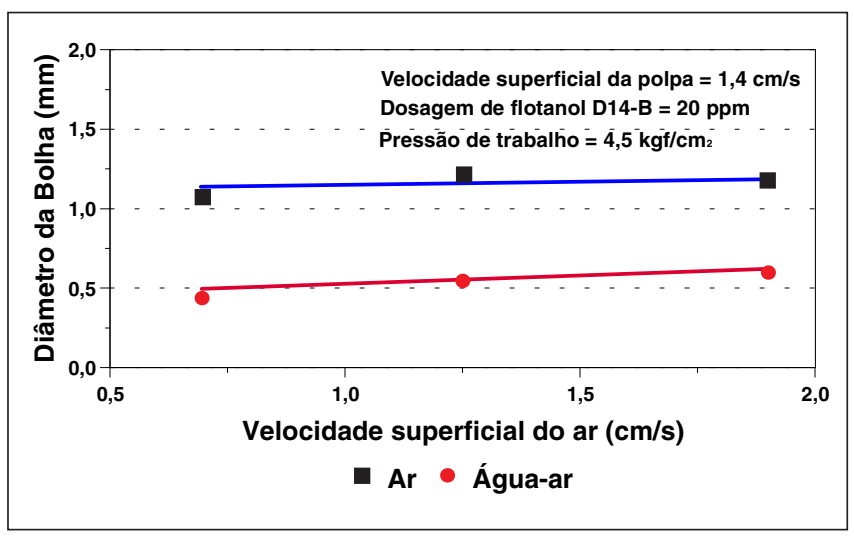

Figura 5 - Efeito da velocidade superficial do ar sobre o tamanho das bolhas.

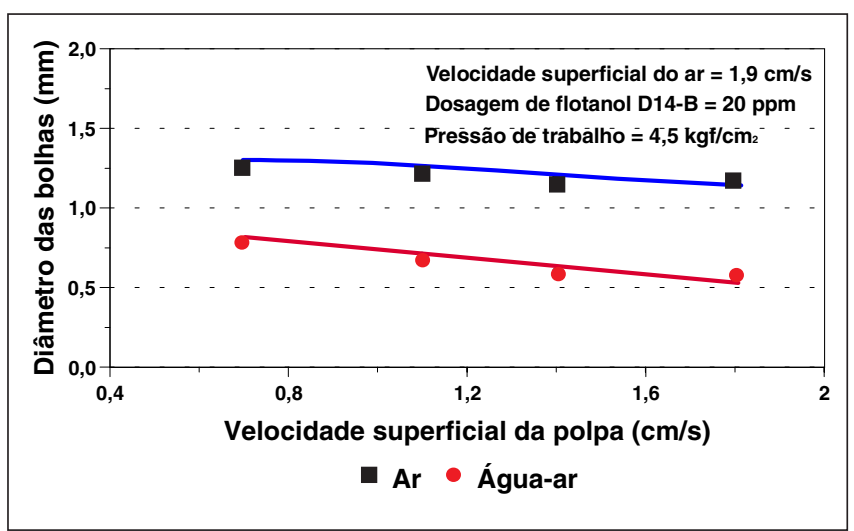

Figura 7 - Efeito da velocidade superficial da polpa sobre o tamanho das bolhas. 


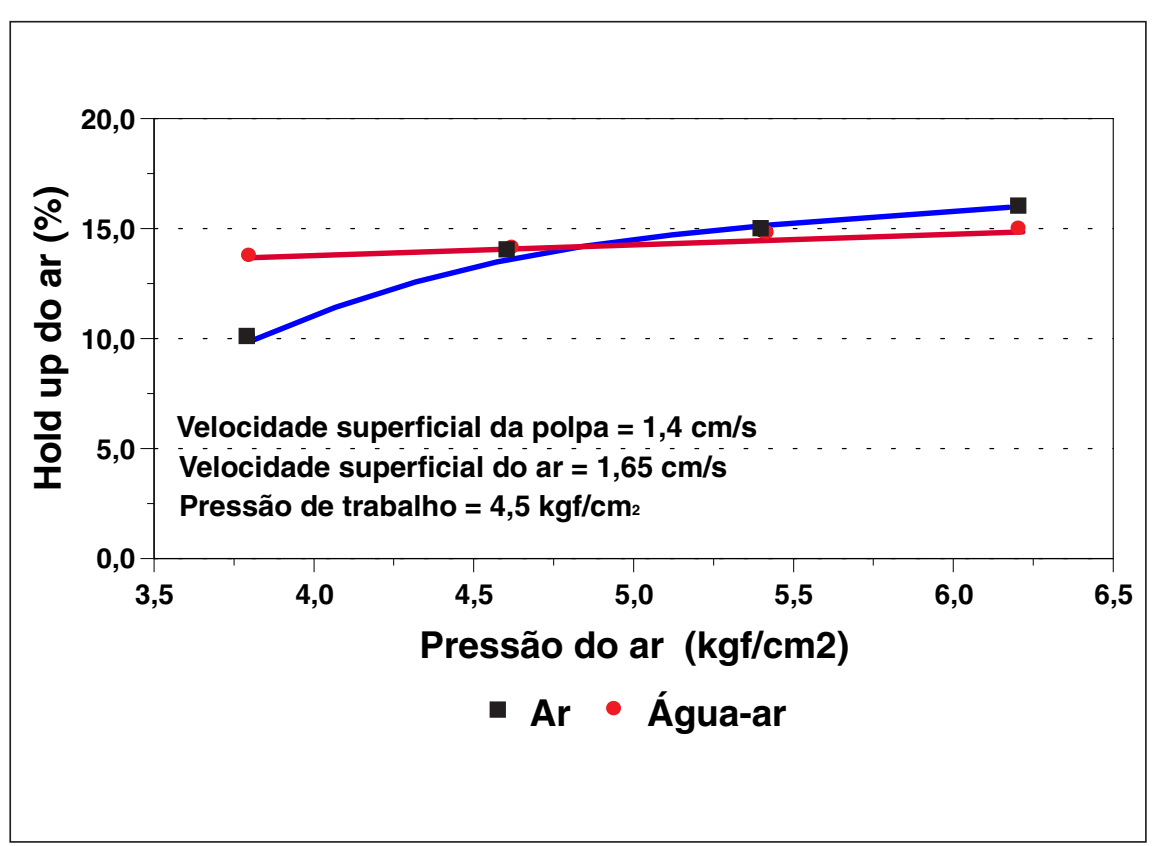

Figura 8 - Efeito da pressão do ar sobre o valor de hold up na coluna.

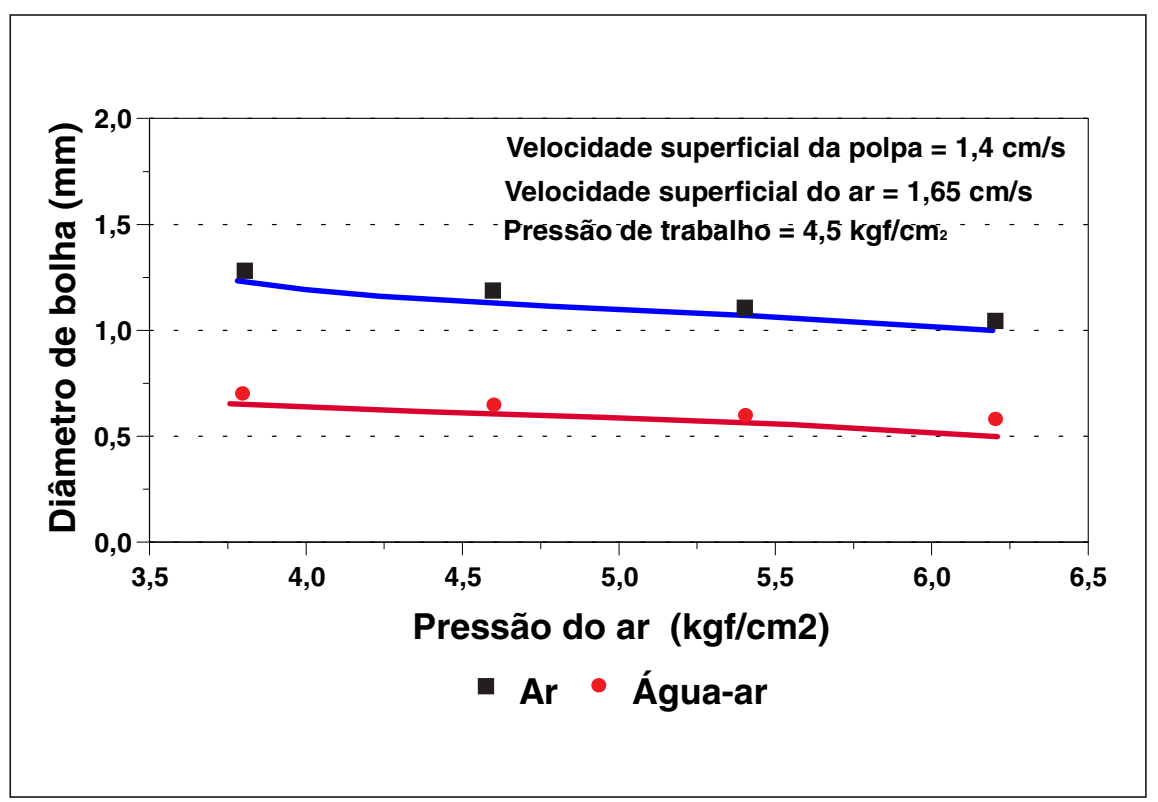

Figura 9 - Efeito da pressão do ar sobre o diâmetro das bolhas.

\section{Conclusões}

Os resultados obtidos mostram que, nas condições usuais de operação das colunas de flotação, a utilização do aerador tipo água-ar acarreta a geração de bolhas com diâmetro médio menor que os obtidos com o aerador do tipo ar.

Considerando o sistema bifásico, ou seja, água e ar, para ambos os aeradores, o diâmetro médio das bolhas de ar na seção de recuperação da coluna foi decrescente com o aumento nos valores das variáveis: dosagem de espumante, velocidade superficial da polpa, pressão de operação dos aeradores, e ligeiramente decrescente com o aumento na velocidade superficial do ar.

Assim, nas colunas de flotação industriais aplicadas à concentração de minérios onde o controle do tamanho e uniformidade de distribuição das bolhas de ar sejam fatores decisivos para obtenção de um bom desempenho metalúrgico, o aerador tipo água-ar é o mais recomendado.

\section{Referências}

\section{Bibliográficas}

DOBBY, G. S., YIANATOS, J. B., FINCH, J. A. Estimation of bubble diameter in flotation columns from drift flux analysis. Canadian Metallurgical Quarterly. v. 27, n. 2. p. 85-90, 1986.

FINCH, J. A., DOBBY, G. S. Column flotation. Pergamon Press. 1990.

KELLY, E. G., SPOTTISWOOD, D. J. Introduction to mineral processing. John Wiley \& Sons, 1982.

LUZ, A. B., POSSA, M. V., ALMEIDA, S. L. Tratamento de minérios, 2a edição revisada e ampliada. Capítulo 10 - CETEM - Centro de Tecnologia Mineral - CNPq/MCT, 1998.

WHEELER, D. A. Column flotation - the original column. McGill University Seminar, May 1986.

Artigo recebido em 31/10/2002 e aprovado em 11/09/2003.

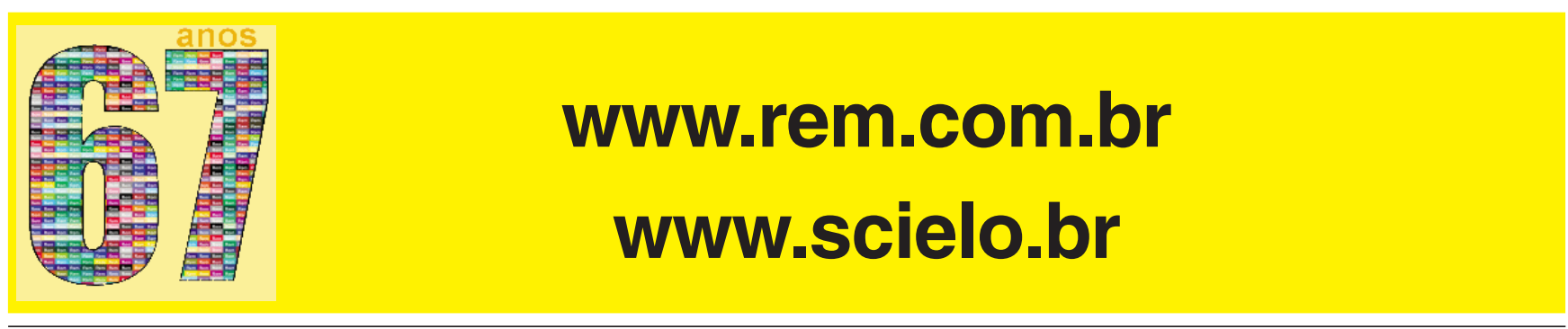

200 REM: R. Esc. Minas, Ouro Preto, 56(3): 195-200, jul. set. 2003 